\title{
International Ophthalmology \\ Effect of nutritional supplement based on melatonin on the intraocular pressure in normotensive subjects \\ --Manuscript Draft--
}

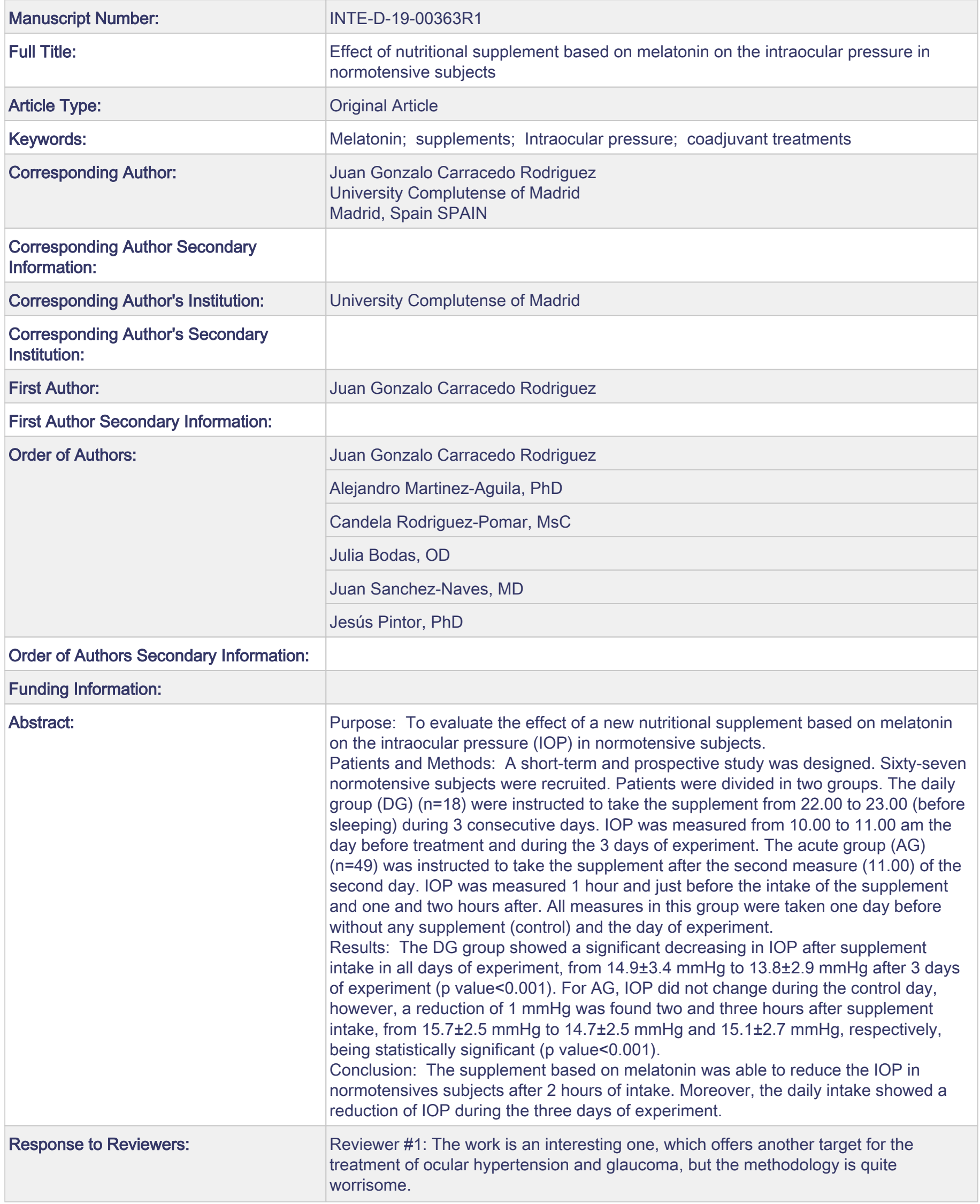


The findings from this study would have been objective, if the study were properly designed.

The study design would have been a controlled randomised clinical trial, to elucidate the objective effect of melatonin on IOP.

The purpose of this study was to corroborate the effect of melatonin on IOP of normotensive patients that was achieve many years ago by Samples (Effect of melatonin on intraocular pressure, Samples JR, Krause G, Lewy AJ. Curr Eye Res. $1988 \mathrm{Jul} ; 7(7): 649-53)$, and to probe that melatonin not only decrease IOP in an acute way, but also that this effect could be detected after the acute effect finished.

Although there is no placebo group in this study, there is a basal IOP from all patients at the same time points studied, that has a better correlation with the final IOP than comparing two different groups.

It would have been age and sex-matched, with equal number of participants in each arm, who were not statistically different.

While there is a difference between male and female patients (1.8:1 ratio), there is no correlation between gender and IOP excluding pregnancy and breastfeeding (Gender difference in the pathophysiology and treatment of glaucoma. Tehrani S Curr Eye Res. 2015 Feb;40(2):191-200) and there is no report about this possibility in melatonin's effect.

There should have been a control group, who were given placebo.

Although there is no placebo group in this study, there is a basal IOP from all patients at the same time points studied, that has a better correlation with the final IOP than comparing two different groups. That is why we decided to make crossed study better than a parallel one with placebo group.

Considering the diurnal / nocturnal variations in IOP, the exact time of ingestion of the supplement for both groups should have been specified.

All patients from AG received the supplement from 10:00 to 11:00 with measures the day before and the same day.

Patients from DG received the supplement at 22:00 the 3 days.

Both sentences has been added to the text in abstract and at line 68 , in order to clarify that.

IOP values from the placebo group would have ruled out the diurnal / nocturnal variations and highlighted the significance of the values in DG and AG.

We know that there is a variation in IOP during all day, and that is why we have decided to take the intraocular pressure at the same time during both days (the control one and the treated one). With these two days, we are able to compare which is the normal diurnal variation and which is made by melatonin supplement.

On the DG, there is also measures from the first day without any treatment, which can be also considered as basal values. In order to compare the results, the two first measures of the first day (10:00 and 11:00) were used as basal values and the same measures were made on the others to avoid day variations.

Why was it only one-time ingestion by AG and not for 3 mornings?

Because the main outcome of this group was to assess its acute effect which last from 2 to 4 hours, not the daily effect.

Taking into account that the same patients could not be used for both experiments, we decided to split them and check separately the acute effect and the daily or chronic treatment.

Why were the participants not examined to confirm the absence of normal pressure glaucoma?

Patients were examined and consulted about this point on its anamnesis to ensure there were no ophthalmic diseases like glaucoma or any retinal one. We have included in the text at line 54. 


\section{Effect of nutritional supplement based on melatonin on} the intraocular pressure in normotensive subjects

Gonzalo Carracedo' PhD; Alejandro Martinez-Aguila² PhD; Candela RodriguezPomar $^{2}$ MsC; Julia Bodas ${ }^{1}$ OD; Juan Sanchez-Naves ${ }^{1}$ MD; Jesús Pintor PhD ${ }^{1}$

$7 \quad{ }^{1}$ Ocupharm Diagnostics group research, Faculty of Optic and Optometry, Universidad

8 Complutense de Madrid, Madrid, Spain

$9 \quad{ }^{2}$ Avizor S.A., Madrid, Spain

Conflict of interest: Gonzalo Carracedo declares that he has no conflict of interest;

Alejandro Martinez-Aguila that he is employee of Avizor SA; Candela Rodriguez-Pomar declares that she is employee of Avizor SA; Julia Bodas declares that she has no conflict of interest; Juan Sanchez declares that he has no conflict of interest; Jesus Pintor declares that he has no conflict of interest

Corresponding Author:

Gonzalo Carracedo Rodríguez

Spain

e-mail: jgcarrac@ucm.es 


\section{ABSTRACT}

2 Purpose: To evaluate the effect of a new nutritional supplement based on melatonin 3 on the intraocular pressure (IOP) in normotensive subjects.

4 Patients and Methods: A short-term and prospective study was designed. Sixty-seven 5 normotensive subjects were recruited. Patients were divided in two groups. The daily 6 group (DG) $(n=18)$ were instructed to take the supplement at night from 22.00 to 23.00 7 (before sleeping) during 3 consecutive days. IOP was measured atfrom 10.00 to 11.00 8 am the day before treatment and during the 3 days of experiment. The acute group 9 (AG) ( $n=49)$ was instructed to take the supplement after the second measure (11.00) of 10 the second dayin the morning only one day. IOP was measured 1 hour and just before

11 the intake of the supplement and one and two hours after. All measures in this group 12 were taken one day after before without any supplement (control) and the day of 13 experiment.

14 -Results: The DG group showed a significant decreasing in IOP after supplement 15 intake in all days of experiment, from $14.9 \pm 3.4 \mathrm{mmHg}$ to $13.8 \pm 2.9 \mathrm{mmHg}$ after 3 days 16 of experiment ( $p$ value $<0.001$ ). For $A G$, IOP did not change during the control day, 17 however, a reduction of $1 \mathrm{mmHg}$ was found two and three hours after supplement 18 intake, from $15.7 \pm 2.5 \mathrm{mmHg}$ to $14.7 \pm 2.5 \mathrm{mmHg}$ and $15.1 \pm 2.7 \mathrm{mmHg}$, respectively, 19 being statistically significant ( $p$ value $<0.001)$.

20 Conclusion: The supplement based on melatonin was able to reduce the IOP in 21 normotensives subjects after 2 hours of intake. Moreover, the daily intake showed a 22 reduction of IOP during allthe three days of experiment.

23 Keywords: Melatonin; supplements; Intraocular pressure; coadjuvant treatments 
24 Ocular hypertension is an anomalous physiological situation related to the appearance of ocular pathologies such as glaucoma. The relationship between ocular hypertension and glaucomatous pathology has been well known for many years. Even today, ocular hypertension is still considered the most important risk factor for the development of this disease [1].

30 Melatonin is an neurohormone that not only is produced by the pineal gland but is also synthesized and released by some ocular structures such as the retina, ciliary body or the lens [2]. Osborne described that melatonin is involved in the control of the aqueous humour dynamics[3]. In this sense, studies performed in animal models suggested that melatonin and its analogues can reduce IOP in normotensive and hypertensive eyes[4]. This control of IOP carried out by melatonin has invited to use of this compound and its analogues for the treatment of ocular hypertension and glaucoma[5]. There are some studies in humans that show the similar effect after melatonin intake [6-8].

A new nutritional supplement as coadjuvant treatment has been developed, based on melatonin to reduce the IOP and other components to improve the other three fundamental concerns of glaucomatous pathology: the elimination of free radicals, the protection and reinforcement of the nervous system and the increase in retinal blood supply $[9,10]$. The objective of this study was to evaluate the possible acute and daily effect of a new nutritional supplement based on melatonin on the intraocular pressure (IOP) in normotensive subjects.

A short-term, prospective and randomized study has been performed. Sixty-seven normotensives subjects were recruited from the Optometry Clinic of the Faculty of Optics and optometry (University Complutense of Madrid, Spain). -Mean age was 
52 beginning the study, the risks and benefits of the treatment were explained, and 53 informed consent was obtained from all subjects. Once included, each subject was 54 examined by ophthalmologist in order to detect any eye disease not mentioned before. 55 Participants were free to leave the study at any time. The study was conducted in compliance with good clinical practice guidelines, institutional review board regulations

57 (IRB number approval: $18.444 \mathrm{O}-\mathrm{P}$ ) and the tenets of the Declaration of Helsinki 58 revised in 2013 [11].

60 A specific nutritional supplement for glaucoma was evaluated (Visaid Press; Avizor SA; 61 Madrid, Spain). This supplement is based on melatonin (1 $\mathrm{mg})$, due its antioxidant and hypotensor efficacy [10], and accompanied by vitamin B6 for its properties acting on 63 the nervous system [12], anthocyanins, as blood flow promoter [13], Docosahexaenoic 64 acid (DHA), Eicosapentaenoic acid (EPA) and manganese. The supplements are 65 administered as a gel pill. The manufacturer intake recommendation is one pill at night.

67 Subjects were randomly divided in two groups. The daily group (DG) $(n=18)$ were 68 instructed to take the supplement from 22.00 to 23.00 , before going to sleep ${ }_{2}$ during 3 69 consecutive days. IOP was measured at 10.00 and 11.00 am one day before, as 70 baseline, and during the 3 days of experiment at the same time points. The acute 71 group (AG) ( $n=49)$ was instructed to take the supplement at the morning only during 72 one day. IOP was measured 1 hour and just before to take the supplement and one 73 and two hours after. All measures in this group were taken one day after-before without 74 any supplement (control) and the day of experiment by the same experienced examiners (C.R and J.B.).

77 For IOP measurement, VX-130 platform (Luneau, Technologies, Chartres, France) was used. The VX130 is a multi-diagnostic platform that combines non-contact tonometer (air puff based) with Hartmann-Shack based autorefraction and Placido-disk and 
80 Scheimpflug based corneal-topography. Three measurements were taken on each 81 subject and the average of the readings was recorded as the final IOP. The mean of 82 measures at 10.00 and 11.00 was considered as basal value for each day. During the measurement, subjects were asked to keep the eye open and fixate to the light stimulus of the device.

85

Data were analysed by statistical package SPSS version 22.0 for Windows (SPSS, Inc., Chicago, IL). The values presented are the means \pm SD of the experiments performed. Parametric tests were used to compare the studied groups. Differences between baseline and after supplement intake in both groups were estimated by the Student - $\mathrm{t}$ test for related samples. One-way ANOVA for related samples were used to evaluate the trend of IOP in DG group. $\mathrm{P}<0.05$ was considered statistically significant.

The DG group showed a significant decreasing in IOP after supplement intake in all days of experiment compared with baseline, being $14.9 \pm 3.4 \mathrm{mmHg}$ for baseline and $14.1 \pm 2.7 \mathrm{mmHg}, 13.6 \pm 2.7 \mathrm{mmHg}$ and $13.8 \pm 2.9 \mathrm{mmHg}$ after 24 hours, 48 hours and 72 hours of experiment, respectively ( $p$ value $<0.001$; Student t-test for related samples). Moreover, the IOP trend during the complete experiment was to decrease, being statistically significant ( $p$ value $<0.05$; One-way ANOVA for related) Figure 1.

100 Regarding AG group, IOP did not change during the control day, being $15.7 \pm 2.4$ $101 \mathrm{mmHg}$ before to supplement intake and $15.6 \pm 2.5 \mathrm{mmHg}$ and $15.6 \pm 2.6 \mathrm{mmHg}$ after 102 two and three hours, respectively ( $p$ value $>0.05$; Student t-test for related samples). 103 However, a reduction of $1 \mathrm{mmHg}$ was found 2 hours after supplement intake, from $10415.7 \pm 2.5 \mathrm{mmHg}$ to $14.7 \pm 2.5 \mathrm{mmHg}$, being statistically significant ( $p$ value $<0.05$;

105 Student t-test for related samples). After three hours, IOP followed being statistically 106 inferior than baseline, with a mean value of $15.1 \pm 2.7 \mathrm{mmHg}$ ( $p$ value $<0.05$; Student $t-$ 107 test for related samples). Figure 2. 
109 The nutritional supplement based on melatonin decreased IOP in normotensive

110 subjects in both experiments performed. A single dose reduces IOP around $7 \%$ after

111 two hours of its ingest and the consecutive intake for three days was able to reduce it

112 around $9 \%$.

114 The effect of melatonin or analogues over IOP has been previously described in

115 humans by oral intake. Samples et al. found that oral melatonin administration (500

$116 \mathrm{mg}$ ) reduced IOP in normal subject around 10\% [8]. Pescosolido et al. found that

117 agomelatine, a melatonin analogue, was able to reduce IOP around $30 \%$ after oral

118 treatment in a group of primary open angle glaucoma patients. These patients were

119 treated with $25 \mathrm{mg}$ of agomelatine for psychiatric conditions [6]. Another study,

120 published in 2009, has demonstrated IOP reduction when patients that underwent

121 cataract surgery were treated with melatonin. These patients were treated with $10 \mathrm{mg}$

122 of melatonin and IOP decreased significantly from $17.9 \pm 0.9$ to $14.2 \pm 1.0 \mathrm{mmHg}$ after

123 premedication and to $13.8 \pm 1.1 \mathrm{mmHg}$ during surgery [7]. -Both studies found an IOP

124 reduction higher that the present trial probably due to the different concentration

125 ingested and to the initial patient IOP.

127 The main limitation of the study is that there is no placebo group, but results are

128 compared to the measures of the same patient at the same time points in the previous

129 day. It should also be considered that the use-measures are performed efwith non-

130 contact tonometer based on air-puff. Some studies found that the non-contact

131 tonometry gives higher results than Goldman tonometry, especially in adult subjects

132 and hypertensive patients [14]. But, the repeatability of non-contact tonometry has

133 been previously described in normotensive population [15]. -lt is-should be important to

134 perform the same study with a placebo group and Goldman or Perkins tonometry in 
135 both normotensives and hypertensive patients to corroborate the outcomes of this 136 study.

138 The nutritional supplement evaluated is composed for other components with 139 interesting features for glaucoma as it has been mentioned in methods section and,

140 taking account the outcomes found in the current study, it would be interesting to

141 design and develop some long-term studies to evaluate the supplement effect in retina 142 protection.

144 In conclusion, the nutritional supplement based on melatonin seems to decrease the 145 IOP in normotensive subjects. These outcomes indicate that melatonin 146 supplementation could be a coadjuvant treatment of glaucoma. Nevertheless, it is 147 mandatory to confirm this role in glaucomatous patients compared with placebo group 148 and with more studies about IOP changes after supplement intake.

\section{Compliance with Ethical Standards:}

151 Funding statement: The authors do not have any financial interest on the materials

152 and instruments used in this study and this study was not funded by third-parties.

153 Ethical approval: All procedures performed in studies involving human participants

154 were in accordance with the ethical standards of the institutional and/or national research committee and with the 1964 Helsinki declaration and its later amendments or comparable ethical standards. 


\section{REFERENCES}

166 [1] R.J. Casson, G. Chidlow, J.P. Wood, J.G. Crowston, I. Goldberg, Definition of

167 glaucoma: clinical and experimental concepts, Clin Exp Ophthalmol 40(4) (2012) 3411689

169 [2] P. Alarma-Estrany, J. Pintor, Melatonin receptors in the eye: location, second 170 messengers and role in ocular physiology, Pharmacol Ther 113(3) (2007) 507-22.

171 [3] N.N. Osborne, Serotonin and melatonin in the iris/ciliary processes and their 172 involvement in intraocular pressure, Acta Neurobiol Exp (Wars) 54 Suppl (1994) 57-64.

173 [4] A. Martinez-Aguila, B. Fonseca, A. Bergua, J. Pintor, Melatonin analogue 174 agomelatine reduces rabbit's intraocular pressure in normotensive and hypertensive 175 conditions, Eur J Pharmacol 701(1-3) (2013) 213-7.

176 [5] A. Mediero, P. Alarma-Estrany, J. Pintor, New treatments for ocular hypertension, 177 Auton Neurosci 147(1-2) (2009) 14-9.

178 [6] N. Pescosolido, V. Gatto, A. Stefanucci, D. Rusciano, Oral treatment with the 179 melatonin agonist agomelatine lowers the intraocular pressure of glaucoma patients, 180 Ophthalmic Physiol Opt 35(2) (2015) 201-5.

181 [7] S.A. Ismail, H.A. Mowafi, Melatonin provides anxiolysis, enhances analgesia, 182 decreases intraocular pressure, and promotes better operating conditions during 183 cataract surgery under topical anesthesia, Anesth Analg 108(4) (2009) 1146-51.

184 [8] J.R. Samples, G. Krause, A.J. Lewy, Effect of melatonin on intraocular pressure, 185 Curr Eye Res 7(7) (1988) 649-53.

186 [9] I. Majsterek, K. Malinowska, M. Stanczyk, M. Kowalski, J. Blaszczyk, A.K. 187 Kurowska, A. Kaminska, J. Szaflik, J.P. Szaflik, Evaluation of oxidative stress markers 188 in pathogenesis of primary open-angle glaucoma, Exp Mol Pathol 90(2) (2011) 231-7. 
189 [10] D.X. Tan, L.C. Manchester, R. Hardeland, S. Lopez-Burillo, J.C. Mayo, R.M. Sainz,

190 R.J. Reiter, Melatonin: a hormone, a tissue factor, an autocoid, a paracoid, and an

191 antioxidant vitamin, J Pineal Res 34(1) (2003) 75-8.

192 [11] A. World Medical, World Medical Association Declaration of Helsinki: ethical

193 principles for medical research involving human subjects, JAMA 310(20) (2013) 2191-

1944.

195 [12] H. Hellmann, S. Mooney, Vitamin B6: a molecule for human health?, Molecules 196 15(1) (2010) 442-59.

197 [13] H. Ohguro, I. Ohguro, S. Yagi, Effects of black currant anthocyanins on intraocular 198 pressure in healthy volunteers and patients with glaucoma, J Ocul Pharmacol Ther $19929(1)(2013) 61-7$.

200 [14] S. Sudesh, M.J. Moseley, J.R. Thompson, Accuracy of Goldmann tonometry in 201 clinical practice, Acta ophthalmologica 71(2) (1993) 185-8.

202 [15] K.C. Ogbuehi, T.M. Almubrad, Accuracy and reliability of the Keeler Pulsair 203 EasyEye non-contact tonometer, Optom Vis Sci 85(1) (2008) 61-6. 


\section{FIGURES LEGENDS}

211

212 FIGURE 1. Effect on IOP during the three days of experiment in daily group (DG).

$213 \mathrm{n}=18$. It is represented the mean and standard error of the mean (SEM). * $p$

214 value<0.05; after intake vs before intake (PRE) (Student t-test for related samples).

215

216

217 FIGURE 2. Effect on IOP after two and three hours of a unique melatonin supplement

218 intake in acute group (AG). $n=49$. It is represented the mean and standard error of the

219 mean (SEM). * $\mathrm{p}$ value<0.05; after intake vs before intake (PRE) (Student t-test for 220 related samples). 


\section{ABSTRACT}

2 Purpose: To evaluate the effect of a new nutritional supplement based on melatonin 3 on the intraocular pressure (IOP) in normotensive subjects.

4 Patients and Methods: A short-term and prospective study was designed. Sixty-seven 5 normotensive subjects were recruited. Patients were divided in two groups. The daily 6 group (DG) $(n=18)$ were instructed to take the supplement from 22.00 to 23.00 (before 7 sleeping) during 3 consecutive days. IOP was measured from 10.00 to 11.00 am the 8 day before treatment and during the 3 days of experiment. The acute group (AG) $9(n=49)$ was instructed to take the supplement after the second measure (11.00) of the 10 second day. IOP was measured 1 hour and just before the intake of the supplement 11 and one and two hours after. All measures in this group were taken one day before 12 without any supplement (control) and the day of experiment.

13 Results: The DG group showed a significant decreasing in IOP after supplement 14 intake in all days of experiment, from $14.9 \pm 3.4 \mathrm{mmHg}$ to $13.8 \pm 2.9 \mathrm{mmHg}$ after 3 days 15 of experiment ( $p$ value $<0.001$ ). For $A G$, IOP did not change during the control day, 16 however, a reduction of $1 \mathrm{mmHg}$ was found two and three hours after supplement 17 intake, from $15.7 \pm 2.5 \mathrm{mmHg}$ to $14.7 \pm 2.5 \mathrm{mmHg}$ and $15.1 \pm 2.7 \mathrm{mmHg}$, respectively, 18 being statistically significant ( $p$ value $<0.001)$.

19 Conclusion: The supplement based on melatonin was able to reduce the IOP in 20 normotensives subjects after 2 hours of intake. Moreover, the daily intake showed a 21 reduction of IOP during the three days of experiment.

22 Keywords: Melatonin; supplements; Intraocular pressure; coadjuvant treatments 
23 Ocular hypertension is an anomalous physiological situation related to the appearance

24 of ocular pathologies such as glaucoma. The relationship between ocular hypertension and glaucomatous pathology has been well known for many years. Even today, ocular hypertension is still considered the most important risk factor for the development of

27 this disease [1].

Melatonin is an neurohormone that not only is produced by the pineal gland but is also synthesized and released by some ocular structures such as the retina, ciliary body or the lens [2]. Osborne described that melatonin is involved in the control of the aqueous humour dynamics[3]. In this sense, studies performed in animal models suggested that melatonin and its analogues can reduce IOP in normotensive and hypertensive eyes[4]. This control of IOP carried out by melatonin has invited to use of this compound and its analogues for the treatment of ocular hypertension and glaucoma[5]. There are some studies in humans that show the similar effect after melatonin intake [6-8].

A new nutritional supplement as coadjuvant treatment has been developed, based on melatonin to reduce the IOP and other components to improve the other three

41 fundamental concerns of glaucomatous pathology: the elimination of free radicals, the

42 protection and reinforcement of the nervous system and the increase in retinal blood supply $[9,10]$. The objective of this study was to evaluate the possible acute and daily effect of a new nutritional supplement based on melatonin on the intraocular pressure (IOP) in normotensive subjects.

47 A short-term, prospective and randomized study has been performed. Sixty-seven 48 normotensives subjects were recruited from the Optometry Clinic of the Faculty of 49 Optics and optometry (University Complutense of Madrid, Spain). Mean age was 42.64 $50 \pm 9.01$ years (range 23 to 54 years), being 43 men and 24 women. Before beginning 
51 the study, the risks and benefits of the treatment were explained, and informed consent was obtained from all subjects. Once included, each subject was examined by ophthalmologist in order to detect any eye disease not mentioned before. Participants were free to leave the study at any time. The study was conducted in compliance with good clinical practice guidelines, institutional review board regulations (IRB number approval: 18.444 O-P) and the tenets of the Declaration of Helsinki revised in 2013 [11].

A specific nutritional supplement for glaucoma was evaluated (Visaid Press; Avizor SA; Madrid, Spain). This supplement is based on melatonin (1 mg), due its antioxidant and hypotensor efficacy [10], and accompanied by vitamin B6 for its properties acting on the nervous system [12], anthocyanins, as blood flow promoter [13], Docosahexaenoic acid (DHA), Eicosapentaenoic acid (EPA) and manganese. The supplements are administered as a gel pill. The manufacturer intake recommendation is one pill at night.

Subjects were randomly divided in two groups. The daily group (DG) $(n=18)$ were instructed to take the supplement from 22.00 to 23.00 , before going to sleep, during 3 consecutive days. IOP was measured at 10.00 and 11.00 am one day before, as baseline, and during the 3 days of experiment at the same time points. The acute group (AG) ( $n=49)$ was instructed to take the supplement at the morning only during one day. IOP was measured 1 hour and just before to take the supplement and one and two hours after. All measures in this group were taken one day before without any supplement (control) and the day of experiment by the same experienced examiners (C.R and J.B.).

For IOP measurement, VX-130 platform (Luneau, Technologies, Chartres, France) was used. The VX130 is a multi-diagnostic platform that combines non-contact tonometer (air puff based) with Hartmann-Shack based autorefraction and Placido-disk and 
Scheimpflug based corneal-topography. Three measurements were taken on each subject and the average of the readings was recorded as the final IOP. The mean of measures at 10.00 and 11.00 was considered as basal value for each day. During the measurement, subjects were asked to keep the eye open and fixate to the light stimulus of the device.

Data were analysed by statistical package SPSS version 22.0 for Windows (SPSS, Inc., Chicago, IL). The values presented are the means \pm SD of the experiments performed. Parametric tests were used to compare the studied groups. Differences between baseline and after supplement intake in both groups were estimated by the Student - $\mathrm{t}$ test for related samples. One-way ANOVA for related samples were used to evaluate the trend of IOP in DG group. $\mathrm{P}<0.05$ was considered statistically significant.

The DG group showed a significant decreasing in IOP after supplement intake in all days of experiment compared with baseline, being $14.9 \pm 3.4 \mathrm{mmHg}$ for baseline and $14.1 \pm 2.7 \mathrm{mmHg}, 13.6 \pm 2.7 \mathrm{mmHg}$ and $13.8 \pm 2.9 \mathrm{mmHg}$ after 24 hours, 48 hours and 72 hours of experiment, respectively ( $p$ value $<0.001$; Student t-test for related samples). Moreover, the IOP trend during the complete experiment was to decrease, being statistically significant ( $p$ value $<0.05$; One-way ANOVA for related) Figure 1.

Regarding AG group, IOP did not change during the control day, being $15.7 \pm 2.4$ $\mathrm{mmHg}$ before to supplement intake and $15.6 \pm 2.5 \mathrm{mmHg}$ and $15.6 \pm 2.6 \mathrm{mmHg}$ after two and three hours, respectively ( $p$ value $>0.05$; Student t-test for related samples). However, a reduction of $1 \mathrm{mmHg}$ was found 2 hours after supplement intake, from

$10315.7 \pm 2.5 \mathrm{mmHg}$ to $14.7 \pm 2.5 \mathrm{mmHg}$, being statistically significant ( $p$ value $<0.05$;

104 Student t-test for related samples). After three hours, IOP followed being statistically 105 inferior than baseline, with a mean value of $15.1 \pm 2.7 \mathrm{mmHg}$ ( $p$ value $<0.05$; Student $t-$ 106 test for related samples). Figure 2. 
108 The nutritional supplement based on melatonin decreased IOP in normotensive

109 subjects in both experiments performed. A single dose reduces IOP around $7 \%$ after

110 two hours of its ingest and the consecutive intake for three days was able to reduce it

111 around $9 \%$.

112

113 The effect of melatonin or analogues over IOP has been previously described in

114 humans by oral intake. Samples et al. found that oral melatonin administration (500

$115 \mathrm{mg}$ ) reduced IOP in normal subject around 10\% [8]. Pescosolido et al. found that

116 agomelatine, a melatonin analogue, was able to reduce IOP around $30 \%$ after oral

117 treatment in a group of primary open angle glaucoma patients. These patients were

118 treated with $25 \mathrm{mg}$ of agomelatine for psychiatric conditions [6]. Another study,

119 published in 2009, has demonstrated IOP reduction when patients that underwent

120 cataract surgery were treated with melatonin. These patients were treated with $10 \mathrm{mg}$

121 of melatonin and IOP decreased significantly from $17.9 \pm 0.9$ to $14.2 \pm 1.0 \mathrm{mmHg}$ after

122 premedication and to $13.8 \pm 1.1 \mathrm{mmHg}$ during surgery [7]. Both studies found an IOP

123 reduction higher that the present trial probably due to the different concentration

124 ingested and to the initial patient IOP.

126 The main limitation of the study is that there is no placebo group, but results are

127 compared to the measures of the same patient at the same time points in the previous

128 day. It should also be considered that measures are performed with non-contact 129 tonometer based on air-puff. Some studies found that the non-contact tonometry gives

130 higher results than Goldman tonometry, especially in adult subjects and hypertensive

131 patients [14]. But, the repeatability of non-contact tonometry has been previously

132 described in normotensive population [15]. It should be important to perform the same

133 study with a placebo group and Goldman or Perkins tonometry in both normotensives

134 and hypertensive patients to corroborate the outcomes of this study. 
136 The nutritional supplement evaluated is composed for other components with

137 interesting features for glaucoma as it has been mentioned in methods section and,

138 taking account the outcomes found in the current study, it would be interesting to

139 design and develop some long-term studies to evaluate the supplement effect in retina

140 protection.

141

142 In conclusion, the nutritional supplement based on melatonin seems to decrease the

143 IOP in normotensive subjects. These outcomes indicate that melatonin

144 supplementation could be a coadjuvant treatment of glaucoma. Nevertheless, it is

145 mandatory to confirm this role in glaucomatous patients compared with placebo group

146 and with more studies about IOP changes after supplement intake.

148 Compliance with Ethical Standards:

149 Funding statement: The authors do not have any financial interest on the materials

150 and instruments used in this study and this study was not funded by third-parties.

151 Ethical approval: All procedures performed in studies involving human participants

152 were in accordance with the ethical standards of the institutional and/or national

153 research committee and with the 1964 Helsinki declaration and its later amendments or 154 comparable ethical standards. 


\section{REFERENCES}

165 [1] R.J. Casson, G. Chidlow, J.P. Wood, J.G. Crowston, I. Goldberg, Definition of 166 glaucoma: clinical and experimental concepts, Clin Exp Ophthalmol 40(4) (2012) 3411679

168 [2] P. Alarma-Estrany, J. Pintor, Melatonin receptors in the eye: location, second 169 messengers and role in ocular physiology, Pharmacol Ther 113(3) (2007) 507-22.

170 [3] N.N. Osborne, Serotonin and melatonin in the iris/ciliary processes and their 171 involvement in intraocular pressure, Acta Neurobiol Exp (Wars) 54 Suppl (1994) 57-64.

172 [4] A. Martinez-Aguila, B. Fonseca, A. Bergua, J. Pintor, Melatonin analogue 173 agomelatine reduces rabbit's intraocular pressure in normotensive and hypertensive 174 conditions, Eur J Pharmacol 701(1-3) (2013) 213-7.

175 [5] A. Mediero, P. Alarma-Estrany, J. Pintor, New treatments for ocular hypertension, 176 Auton Neurosci 147(1-2) (2009) 14-9.

177 [6] N. Pescosolido, V. Gatto, A. Stefanucci, D. Rusciano, Oral treatment with the 178 melatonin agonist agomelatine lowers the intraocular pressure of glaucoma patients, 179 Ophthalmic Physiol Opt 35(2) (2015) 201-5.

180 [7] S.A. Ismail, H.A. Mowafi, Melatonin provides anxiolysis, enhances analgesia, 181 decreases intraocular pressure, and promotes better operating conditions during 182 cataract surgery under topical anesthesia, Anesth Analg 108(4) (2009) 1146-51.

183 [8] J.R. Samples, G. Krause, A.J. Lewy, Effect of melatonin on intraocular pressure, 184 Curr Eye Res 7(7) (1988) 649-53.

185 [9] I. Majsterek, K. Malinowska, M. Stanczyk, M. Kowalski, J. Blaszczyk, A.K. 186 Kurowska, A. Kaminska, J. Szaflik, J.P. Szaflik, Evaluation of oxidative stress markers 187 in pathogenesis of primary open-angle glaucoma, Exp Mol Pathol 90(2) (2011) 231-7. 
188 [10] D.X. Tan, L.C. Manchester, R. Hardeland, S. Lopez-Burillo, J.C. Mayo, R.M. Sainz,

189 R.J. Reiter, Melatonin: a hormone, a tissue factor, an autocoid, a paracoid, and an

190 antioxidant vitamin, J Pineal Res 34(1) (2003) 75-8.

191 [11] A. World Medical, World Medical Association Declaration of Helsinki: ethical

192 principles for medical research involving human subjects, JAMA 310(20) (2013) 2191-

1934.

194 [12] H. Hellmann, S. Mooney, Vitamin B6: a molecule for human health?, Molecules 195 15(1) (2010) 442-59.

196 [13] H. Ohguro, I. Ohguro, S. Yagi, Effects of black currant anthocyanins on intraocular

197 pressure in healthy volunteers and patients with glaucoma, J Ocul Pharmacol Ther $198 \quad 29(1)(2013) 61-7$.

199 [14] S. Sudesh, M.J. Moseley, J.R. Thompson, Accuracy of Goldmann tonometry in 200 clinical practice, Acta ophthalmologica 71(2) (1993) 185-8.

201 [15] K.C. Ogbuehi, T.M. Almubrad, Accuracy and reliability of the Keeler Pulsair 202 EasyEye non-contact tonometer, Optom Vis Sci 85(1) (2008) 61-6. 


\section{FIGURES LEGENDS}

210

211 FIGURE 1. Effect on IOP during the three days of experiment in daily group (DG).

$212 n=18$. It is represented the mean and standard error of the mean (SEM). * $p$

213 value $<0.05$; after intake vs before intake (PRE) (Student t-test for related samples).

216 FIGURE 2. Effect on IOP after two and three hours of a unique melatonin supplement

217 intake in acute group (AG). $n=49$. It is represented the mean and standard error of the

218 mean (SEM). * $\mathrm{p}$ value<0.05; after intake vs before intake (PRE) (Student t-test for 219 related samples). 


\section{FIGURE 1}

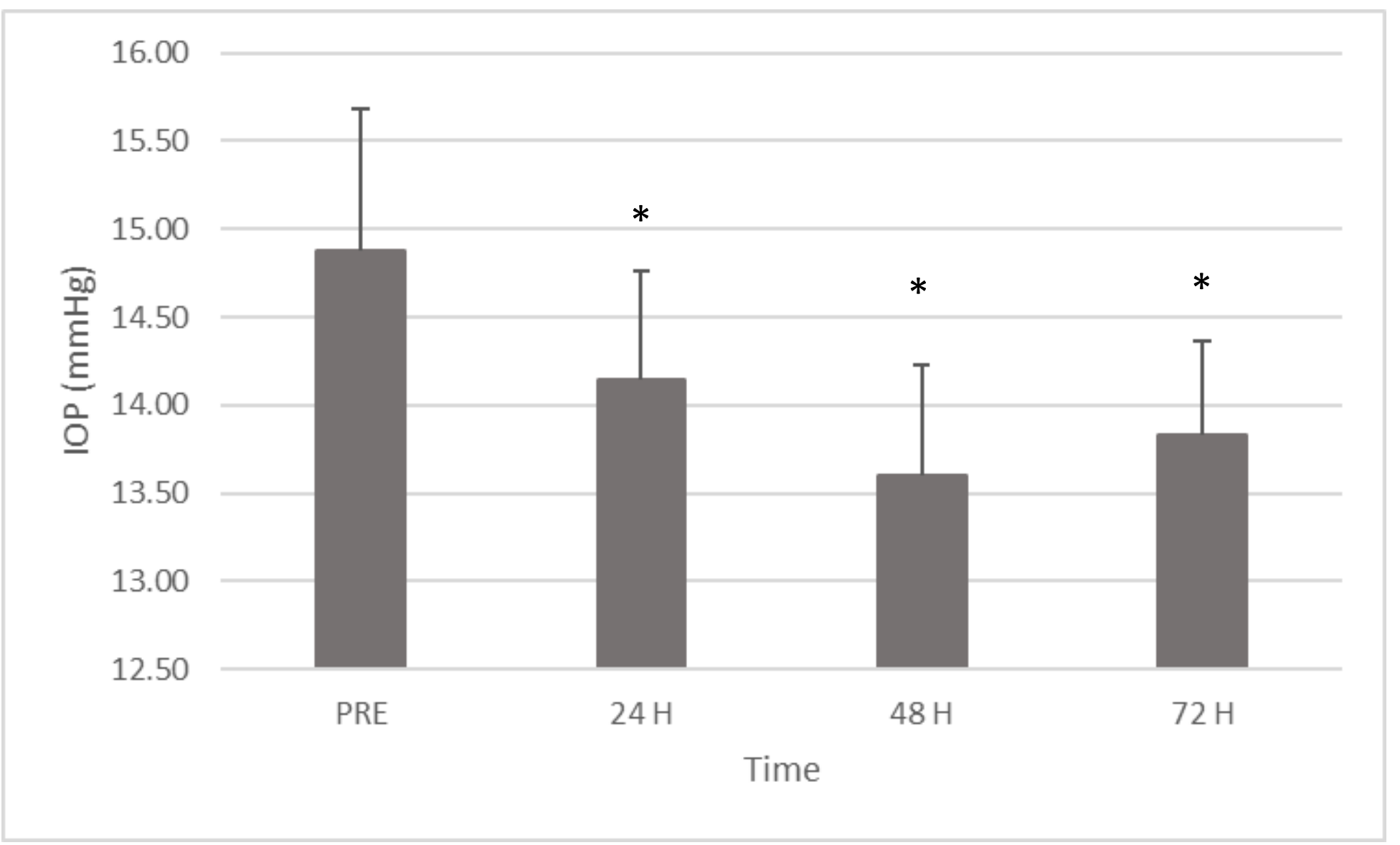




\section{FIGURE 2}

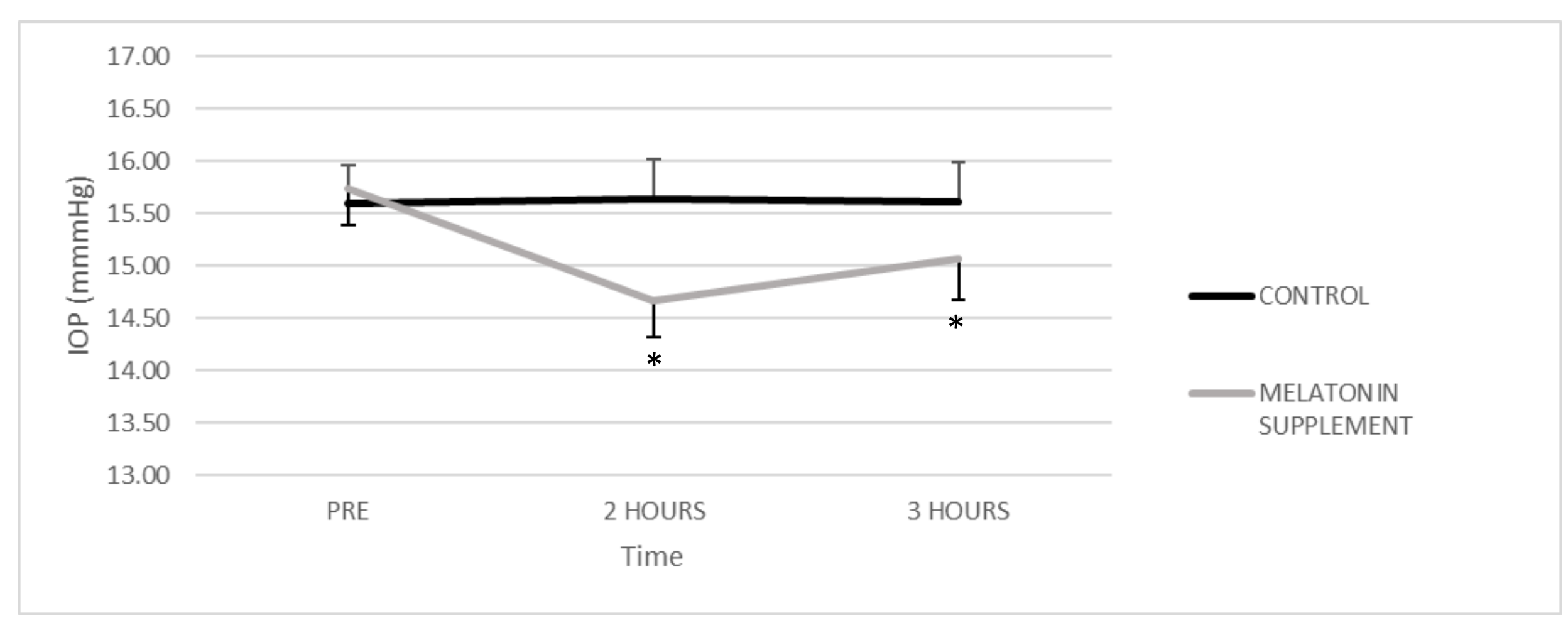

